\title{
Deciphering intratumoral heterogeneity using integrated clonal tracking and single-cell transcriptome analyses
}

Rong Lu ( $\sim$ ronglu@usc.edu )

University of Southern California https://orcid.org/0000-0002-5151-9246

HUMBERTO CONTRERAS-TRUJILLO

University of Southern California

JIYA EERDENG

University of Southern California

SAMIR AKRE

University of Southern California https://orcid.org/0000-0003-1495-6399

DU JIANG

University of Southern California

APARNA JORAPUR

University of Southern California

MARY VERGEL-RODRIGUEZ

University of Southern California

AREEN ANDREASIAN

University of Southern California

LISA HARTON

University of Southern California

Charles Bramlett

University of Southern California

BASIA GALA

University of Southern California

Anna Nogalska

University of Southern California

Lai Chan

Department of Systems Biology, City of Hope Comprehensive Cancer Center

Gang Xiao

Beckman Research Institute of City of Hope

JAE-WOONG LEE

City of Hope Comprehensive Cancer Center

MARKUS MÜSCHEN 
City of Hope Comprehensive Cancer Center

AKIL MERCHANT

Cedars-Sinai Medical Center

\section{Article}

Keywords: leukemia, cellular heterogeneity, single cell transcriptome profiling, clonal tracking

Posted Date: January 8th, 2021

DOl: https://doi.org/10.21203/rs.3.rs-132486/v1

License: (c) (i) This work is licensed under a Creative Commons Attribution 4.0 International License. Read Full License

Version of Record: A version of this preprint was published at Nature Communications on November 11th, 2021. See the published version at https://doi.org/10.1038/s41467-021-26771-1. 


\section{Deciphering intratumoral heterogeneity using integrated clonal tracking and single-cell transcriptome analyses}

Humberto Contreras-Trujillo ${ }^{1}$, Jiya Eerdeng ${ }^{1, \dagger}$, Samir Akre ${ }^{1, \dagger}$, Du Jiang ${ }^{1, \dagger}$, Aparna Jorapur ${ }^{2}$, Mary C. Vergel-Rodriguez ${ }^{1}$, Areen Andreasian ${ }^{1}$, Lisa Harton ${ }^{2}$, Charles S. Bramlett ${ }^{1}$, Basia Gala ${ }^{1}$, Anna Nogalska', Gang Xiao ${ }^{3}$, Jae-Woong Lee ${ }^{3}$, Lai N. Chan ${ }^{3}$, Markus Müschen ${ }^{3}$, Akil A. Merchant ${ }^{4,}$, Rong Lu, ${ }^{1, *}$

1'Department of Stem Cell Biology and Regenerative Medicine, Eli and Edythe Broad Center for Regenerative Medicine and Stem Cell Research, Keck School of Medicine, University of Southern California, Los Angeles, CA 90033, USA.

2Division of Hematology, USC Norris Comprehensive Cancer Center, Keck School of Medicine, University of Southern California, Los Angeles, CA 90033, USA.

${ }^{3}$ Department of Systems Biology, City of Hope Comprehensive Cancer Center, Monrovia, CA 91016, USA.

${ }^{4}$ Blood and Marrow Transplantation Program, Cedars-Sinai Medical Center, Los Angeles, CA 90048, USA.

†These authors contributed equally to this work.

*Correspondence: RongLu@usc.edu (R.L.) and Akil.Merchant@cshs.org (A.A.M.) 


\begin{abstract}
Cellular heterogeneity is a major cause of treatment resistance in cancer. Despite recent advances in single-cell genomic and transcriptomic sequencing, it remains difficult to relate measured molecular profiles to the cellular activities underlying cancer. Here, we present an integrated experimental system that connects single cell gene expression to heterogeneous cancer cell growth, metastasis, and treatment response. Our system integrates single cell transcriptome profiling with DNA barcode based clonal tracking in patient-derived xenograft models. We show that leukemia cells exhibiting unique gene expression signatures respond to different chemotherapies in distinct but consistent manners across multiple mice. In addition, we uncover an unexpected yet common form of leukemia expansion that is spatially confined to the bone marrow of single anatomical sites and driven by cells with distinct gene expression signatures. Our integrated system directly and effectively interrogates the molecular and cellular basis of the intratumoral heterogeneity underlying disease progression and treatment resistance.
\end{abstract}

\title{
Introduction
}

Cancer is a dynamic disease driven by continuous genetic and epigenetic changes ${ }^{1-7}$. The accumulation of these molecular alterations generates tremendous intratumoral heterogeneity ${ }^{1-}$ 4,8,9. Consequently, individual cancer cells differentially proliferate, selectively metastasize, and sporadically escape therapeutic treatment ${ }^{1-4,8,9}$. Cellular heterogeneity has arisen as a major hurdle in cancer treatment ${ }^{10-15}$. Identifying the genes underlying the heterogeneous behaviors of individual cancer cells is critically important to improving the efficacy of cancer treatment.

Recent advances in single cell genomic and transcriptomic sequencing have greatly improved the detection of intratumoral heterogeneity at the molecular level ${ }^{16-22}$. However, it remains difficult to relate the molecular profiles generated by these new technologies to the cellular behaviors underlying disease progression and relapse. Some studies have managed to link a few genes using massive in-depth sequencing to identify naturally occurring genetic mutations that can be used to trace cell clones ${ }^{23-25}$. Although clonal tracking through natural mutations is a powerful technique that does not require any invasive maniputation ${ }^{6,23-30}$, it suffers from a prohibitive cost, low efficiency and complications in clonal comparison that arise from the rarity and randomness of natural mutations. As an alternative to tracking natural mutations, some recent studies have employed synthetic DNA barcodes to simultaneously mark 
individual cancer cells ${ }^{31-33}$. In conjunction with patient-derived xenograft (PDX) models, the use of synthetic DNA barcoding has greatly improved our understanding of the heterogeneous growth and metastasis of cancer cells ${ }^{32-35}$.

In this study, we present an integrated experimental system that directly connects gene expression with cellular behavior at the single cell level by combining synthetic DNA barcode tracking and single cell mRNA sequencing in a PDX model. We have previously demonstrated the high sensitivity and precise quantification of our DNA barcode tracking using hematopoietic stem cells in vivo ${ }^{36-40}$. Here, we adapted the barcode tracking to assay the activities of cancer cells and their gene expression profiles simultaneously in a PDX model xenografted by human B-cell acute lymphoblastic leukemia (B-ALL) samples. In the PDX model, B-ALL cells can autonomously home to their native micro-environment in the bone marrow and can be easily and repeatedly sampled over time to monitor cancer progression and therapeutic response. Using this integrated system, we show that primary B-ALL clones exhibit heterogeneous dynamics during expansion, circulation, and response to chemotherapy. Furthermore, their distinct temporal and spatial clonal dynamics are associated with unique gene expression signatures.

\section{Results}

\section{An integrated experimental system connecting single cell transcriptome and clonal}

tracking. Primary B-ALL cells were genetically barcoded using a GFP-encoding lentiviral vector (Fig. 1a) ${ }^{36,37}$. After barcode labeling, they were transplanted into sub-lethally irradiated NSG or NSG-SGM3 mice. Subsequent clonal tracking assays were performed as previously described ${ }^{36,37}$. Our data show that the barcoded leukemia cells expanded proportionally to nonbarcoded leukemia cells in recipient mice (Fig. 1b and Supplementary Fig. 1), suggesting that barcoded cells are representative of the overall leukemic cell population. As DNA barcodes are inserted into cellular genomes, they are inherited by all descendants of barcoded cells, allowing us to track cellular proliferation and elimination. In this study, a "clone" refers to cells carrying identical barcodes.

To assay single cell gene expression, a fraction of the donor barcoded cells were analyzed by droplet-based single cell transcriptome analyse ${ }^{41,42}$, while the rest of the donor barcoded cells were xenografted into mice to assay cellular activities (Fig. 1a). During the transcriptome assays, cDNAs from individual cells were labeled by distinct cellular indexes. Since the clonal tracking barcodes are transcribed, they can be recovered from the single cell cDNA library together with the cellular indexes. The molecules containing both clonal tracking 
barcodes and cDNA cellular indexes were selectively amplified from the single cell cDNA library and sequenced to attain efficient mapping between single cell gene expression and the clonal activity that was examined in the subsequent recipients (Fig. 1a).

Distinct leukemia clones expand in extramedullary organs. All recipient mice that received cells from three particular patient samples exhibited extramedullary expansion (Fig. 1c-e and Supplementary Fig. 2). Each of these mice exhibited an enlargement of a specific organenlarged kidney, stomach or ovaries-depending on the particular patient sample it received (Fig. 1c-e and Supplementary Fig. 2). We analyzed the human cells from these enlarged organs and found that leukemia clones at the extramedullary sites were often different from those in the hematopoietic tissues, including the peripheral blood (Fig. 1f-h, Supplementary Figs. 3, 4c, and 4f). This demonstrates the existence of clonal selection during extramedullary expansion. We analyzed the gene expression profiles of donor cells, comparing clones that expanded in the ovary with those that did not (Fig. 1i). We identified a largely unknown gene, CMC2 (COX assembly mitochondrial protein 2 homolog), that was expressed at a significantly higher level in clones overrepresented in the ovary (Fig. 1i, j, and Supplementary Fig. 5).

Spatially confined expansion of leukemia clones in the bone marrow. To determine the cellular heterogeneity of leukemia circulation, we compared the clonal composition between different tissues and organs in the primary recipient mice of seven B-ALL samples from five patients. We found that leukemia clones in the blood and spleen were highly correlated in all mice, except for two mice that received the same patient sample (ALL20-M1 and ALL20-M2). In these two mice, one clone dominated the blood, but this clone was not abundant in any other tissues (Fig. 2a and Supplementary Fig. 4a). In a few mice, clones in the bone marrow did not correlate well with those in the blood and the spleen (Fig. 2a). We then compared the bone marrow from different anatomical sites and found that more than half of the primary recipient mice exhibited high clonal correlation while others showed significant differences (Fig. 2b). Mice that received ALL20 and JFK93N samples mostly exhibited considerable differences in clonal correlation (Fig. 2b), suggesting that a cell intrinsic mechanism is responsible for tissue homing patterns.

The clonal disparity of leukemia cells in the bone marrow uncovered by our barcode clonal tracking contradicts the prevalent dogma that leukemia, a "liquid" cancer, uniformly spreads throughout the body (Fig. 2a, b). Furthermore, we discovered that the clonal discrepancy mainly arises from a small number of clones that substantially expand in the bone marrow at single anatomical sites and that do not circulate (Fig. 2c and Supplementary Fig. 4). 
This spatially confined clonal expansion was consistently detected in 15 primary recipient mice that received B-ALL cells from 5 different patients (Fig. 2c and Supplementary Fig. 4a-e). It was not detected in any mice that received one particular patient sample (JFK88N) (Supplementary Fig. 4f), suggesting that it is a cell autonomous characteristic.

To identify the genes associated with the spatially confined clonal expansion in the bone marrow, we performed single cell RNA sequencing analyses of the donor ALL04 cells. We compared the gene expression profiles of the clones that were more abundant in the bone marrow with those that were more abundant in the peripheral blood (Fig. 2d). We found three genes-BTK, DNAJC, and LRIF1 - that were significantly differentially expressed (Fig. 2e and Supplementary Fig. 6). BTK (Bruton's tyrosine kinase) is critical for signal transduction downstream of the pre-B cell receptor (pre-BCR) and functions as a tumor suppressor in B$\mathrm{ALL}^{43,44}$. A BTK-binding molecule, Ibrutinib, is currently being tested in a clinical trial for treating B-ALL (ClinicalTrials.gov Identifier: NCT02997761). Our data here associate the expression of BTK with spatially confined clonal expansion (Fig. 2d-e). The other two genes that we identified, DNAJC and LRIF1, could also be potential therapeutic targets for future studies.

Clonal selection occurs during serial transplantation. Spatially confined clonal expansion was missing in secondary recipient mice (Supplementary Fig. 3), because they are underrepresented in the spleen where donor cells for consecutive transplantation are collected by common convention. This explains why spatially confined clonal expansion was not identified by previous studies as most PDX studies use mice after multiple passages in order to obtain enough biological replicates from limited patient samples. To determine how clonal diversity is influenced by transplantation, we performed serial transplantations of barcoded leukemia clones recovered from the spleen, and compared the clonality of spleen cells between different recipients (Fig. 3a). Comparisons of the primary, secondary and tertiary recipients show that clonal abundances generally change in a monotonic way with passages, where clonal abundances either constantly diminishes or constantly expands (Fig. 3b). Comparing multiple secondary recipient mice that received cells recovered from the same primary recipient (Fig. $3 \mathrm{c}$ ), we found that some clones abundant in primary recipient mice were not detected in secondary recipients (Fig. 3d, e). The clonal correlations between secondary recipients were significantly elevated, suggesting that clonal selection is cell autonomous (Fig. $3 d$, $f$ and Supplementary Fig. 7). The consistent clonality across different secondary recipients allows different therapeutic treatments to be tested on the same set of clones in different mice. 
Combination therapy effectively treats B-ALL in the PDX model. The efficacy of chemotherapy in treating ALL is one of the great successes in medical oncology-transforming a universally fatal disease into a curable one for most children and many adults. One peculiarity of ALL therapy is that multiple cycles of low-dose maintenance therapy after high intensity therapy are necessary for long-term cure (NCCN clinical practice guidelines: Acute Lymphoblastic Leukemia Version 2.2019; Pediatric Acute Lymphoblastic Leukemia Version 1.2020). This complex regimen was derived empirically from decades of methodical clinical research. However, a mechanistic explanation of how intensive and maintenance therapies synergize has never been presented. We hypothesized that intensive and maintenance therapies target different subsets of ALL clones. To test this hypothesis, we transplanted identical B-ALL clones into multiple mice that were subsequently treated with different types of chemotherapy. We used a multi-agent therapy consisting of vincristine, dexamethasone and Lasparaginase for the intensive phase ${ }^{45,46}$ and a single-agent therapy consisting of methotrexate for the maintenance phase, mimicking clinical practice. Experimental mice were randomly assigned to five groups: (i) vehicle control; (ii) combination therapy consisting of short-term intensive therapy followed by prolonged maintenance therapy, designed to approximate the clinical ALL treatment; (iii) short-term intensive therapy; (iv) prolonged intensive therapy; and (v) prolonged maintenance therapy. Maintenance therapy was continuously applied throughout the lives of the mice. Prolonged intensive therapy was applied to the toleration of the mice as assessed by changes to their body weight (Supplementary Fig. 8).

We tested these five regimens on 74 mice that received B-ALL cells from three patients (Fig. 4 and Fig. 5). As expected, mice that received chemotherapy survived longer than those that did not (Fig. 4a). Mice that received the combination therapy consistently survived the longest (Fig. 4a). The survival rate was directly correlated with the fraction of human leukemia cells in the mouse peripheral blood (Fig. $4 \mathrm{~b}$ and Supplementary Fig. 9). Furthermore, intensive therapy effectively and rapidly removed the vast majority of leukemia cells, while maintenance therapy suppressed leukemia growth (Fig. 4b and Supplementary Fig. 9). Mouse body weights dropped quickly in the absence of chemotherapy due to the leukemia burden (Supplementary Fig. 8). The body weight drop was also evident following intensive therapy (Supplementary Fig. 8), consistent with the toxicity of intensive treatment observed in clinical practice. Taken together, our experimental model well recapitulates the key aspects of the two phases of ALL therapy. 


\section{Distinct subsets of B-ALL clones responded differentially to intensive and maintenance}

chemotherapy. We compared leukemia clones before and after various treatments and noted the emergence of previously undetected clones after chemotherapy treatment (Fig. 4c). The emergent clones were abundant at several later time points in multiple tissues (Fig. 4d-e, and Supplementary Figs. 10-13). Their emergence indicates their survival and growth advantages over other clones after chemotherapy. Since mice receiving the same patient sample exhibited similar clonal compositions prior to chemotherapy (Fig. 4c), we were able to determine the effects of different treatments by comparing the clonal composition of mice after the treatments. Identical clones in the peripheral blood, spleen, and bone marrow exhibited consistent chemotherapeutic responses (Fig. 5a). Moreover, some clones exhibited significant consistency in their chemotherapy responses across different mice (Fig. 5b-e and Supplementary Figs. 1417). For example, ALL04 clone 2 consistently responded well to combination therapy, prolonged intensive therapy, and maintenance therapy, but not to short-term intensive therapy. ALL06 clone 4 only responded to combination therapy in all mice. ALL20 clone 1 did not respond to maintenance therapy in any mice, but consistently responded to all treatments that involved an intensive therapy phase (i.e., combination therapy, short-term and prolonged intensive therapy).

While no clone was found to respond better to intensive therapy than to combination therapy (Supplementary Figs. 14-17), several clones from ALL04 and ALL06 responded significantly better to combination therapy than to intensive therapy alone (Fig. $5 \mathrm{c}$ and Supplementary Fig. 17). We performed single cell RNA sequencing analysis on donor B-ALL cells to determine if the clones that exhibited a distinct chemotherapy response share a common gene expression signature prior to treatment. In ALL04, the clones that responded better to combination therapy than to intensive therapy expressed significantly lower levels of EBPL and significantly higher levels of MESDC compared to other clones (Fig. $5 \mathrm{c}$ and Supplementary Fig. 18). In addition, some clones from ALL04 responded significantly better to maintenance therapy than to intensive therapy. Compared to other clones, these clones expressed lower levels of CAPNS1 prior to chemotherapy (Fig. 5d and Supplementary Fig. 19). This finding is in line with a previous study showing that inhibiting CAPNS1 sensitizes prostate cancer cells to methotrexate treatment ${ }^{47}$.

In another patient sample ALL20, some clones responded significantly better to intensive therapy than to maintenance therapy (Fig. 5e). Prior to chemotherapy treatments, these clones expressed higher levels of BTG2, CD38, GTF2A2, ICOSLG, ITGAE and ZRANB2 compared to other clones (Fig. 5e and Supplementary Fig. 20). BTG2 is a tumor suppressor in B-ALL and a 
known target of $\mathrm{p} 53^{48}$. It is upregulated during chemotherapy-mediated apoptosis in cancer cells $^{49,50}$. Our data suggest that the upregulation of BTG2 sensitizes B-ALL clones to intensive chemotherapy treatment. CD38 and ITGAE (CD103) are both activation markers of leukemia, and our data is consistent with the idea that intensive chemotherapy selectively targets the subset of cancer cells that are highly proliferative ${ }^{51}$. Monoclonal antibodies targeting CD38 (daratumumab, isatuximab and MOR202) have been used in many clinical trials for hematopoietic malignancies ${ }^{52}$. CD38 and ITGAE were activated in response to pentostatin ${ }^{51}$, an antimetabolite drug that disrupts nucleic acid synthesis like methotrexate. This is consistent with our finding that clones with higher expression of these genes were less sensitive to methotrexate. ICOSLG has been found to be upregulated in trastuzumab-resistant breast cancer cells ${ }^{53}$, suggesting that it plays a role in therapeutic resistance. Taken together, the data provide original experimental evidence that different subsets of ALL clones are significantly different in their response to intensive and maintenance therapies and possess distinct gene expression signatures prior to chemotherapy.

\section{Relapsed leukemic cells exhibit greater clonal dominance in the PDX model than} chemotherapy-naïve leukemia cells from the same patients. To directly compare primary BALL cells before and after chemotherapy treatment in patients, we collected human B-ALL samples from the diagnosis (naïve) stage and after relapse from each of two patients (Fig. 6a and Supplementary Table 1). The samples from patient JFK93 grew much faster than those from patient JFK88 in vivo (Fig. 6b) and in vitro (Supplementary Fig. 21). Our barcode analysis showed that less than $1 \%$ of donor cells engrafted (Supplementary Fig. 22), highlighting the severe engraftment barrier of the PDX model. In addition, significantly more barcodes were detected in the peripheral blood of mice xenografted with naïve samples than those xenografted with relapsed samples (Fig. 6c, for both JFK88 and JFK93: P value $<0.01$ at the initial time points; $P$ value $<0.001$ across all time points). The clonal diversity was also much higher for the naïve samples compared to the relapsed samples (Fig. 6d), although barcode transduction was abnormally high in one naïve sample under the same experimental condition (Supplementary Fig. 23).

After transplantation, the number of leukemia clones derived from the slow-growing samples of patient JFK88 generally decreased over time (Fig. 6c). This reduction was mainly caused by the elimination of low abundance clones (Fig. 6e-f, and Supplementary Fig. 24). While the growth of high abundance clones was generally stable (Fig. 6f and Supplementary Fig. 24), clonal competition was evident during the later disease stages when the overall growth 
slowed down (Fig. 6b, g). In mice that received the JFK93 naïve sample, exactly one clone outcompeted other clones over time (Fig. 6g). Most mice that received relapse samples were dominated by just one or two clones through all the time points that we examined (Fig. $6 \mathrm{f}$ and Supplementary Fig. 24). These data suggest that PDX models using patient samples derived during relapse are severely prejudiced.

\section{Discussion}

In this study, we directly linked the intratumoral clonal dynamics of individual leukemia cells with their heterogeneous gene expression. We showed that individual leukemia cells exhibit different levels of expansion (Figs. 3 and 6), circulation (Figs. 1 and 2), and chemotherapy response (Figs. 4 and 5). We identified the genes associated with these cellular activities by comparing cells within the same patient samples that exhibited different levels of a cellular activity (Figs. 1, 2 and 5). Approximately half of the identified genes have been previously associated with similar

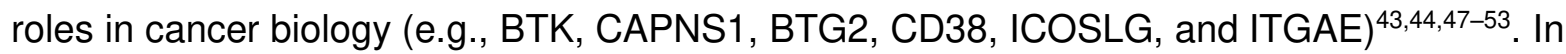
addition, we identified genes of new cancer relevance with regards to leukemia circulation (e.g., LRIF1, DNAJC1, and CMC2) and therapeutic treatment (e.g., EBPL, MESDC2, ZRANB2, GTF2A2) (Figs 1, 2 and 5). These genes could be promising therapeutic targets. Furthermore, our methodology_-identifying genes by assaying intratumoral heterogeneity—can readily be applied to other diseases and biological processes.

Our findings highlight the inherent low engraftment problem of the PDX model (Supplementary Fig. 22). The PDX model is the primary animal model used in cancer studies and drug discovery ${ }^{34,35,54-57}$. It is also regularly used as the final assay prior to clinical trials in humans ${ }^{58-60}$. Here, we show that the engraftment was worse in PDX mice of late passages (Fig. 3 ), which are commonly used in therapeutic development ${ }^{54,61-64}$. Furthermore, when xenografted with patient samples collected after relapse, most PDX mice became dominated by cells that descended from a single patient cell (Fig. 6). As PDX studies generally do not discriminate between samples collected from different disease stages, our data demonstrate that certain samples may be severely biased. The sampling limitations of the PDX model are particularly troubling in the light of the heterogeneity of cancer cells $\mathbf{s}^{1-4,8,9}$.

Our data precisely delineate the heterogenous clonal dynamics of leukemia using robust biological replicate assays that eliminate the influence of rare molecular events, such as random viral integration ${ }^{36-40}$. Using 5 primary patient samples and 15 primary xenograft mice, we showed that dominant clonal expansion in the bone marrow is commonly restricted to confined anatomical sites (Figs. 2 and Supplementary Fig. 4). This surprising finding suggests that bone 
marrow biopsies, widely used for diagnosis ${ }^{65,66}$, may not accurately represent the overall disease condition. Spatially confined leukemia clones expand aggressively without circulating (Figs. 2 and Supplementary Fig. 4). As they are not collected for subsequent transplantation, they were missed by prior studies that relied on xenografts of later passages. In addition, we provide a direct mechanistic explanation for the famed success of combination therapy in ALL treatment ${ }^{67,68}$. We show that ALL clones exhibit consistent and distinct responses to intensive and maintenance chemotherapy across different mice (Figs. 4-5), supporting the hypothesis that intensive and maintenance therapies synergize by targeting different subsets of ALL clones. Our study can help improve treatments for other types of cancer by offering a new strategy to identify and characterize treatment-resistant cells through deciphering intratumoral heterogeneity.

\section{Methods}

Human cells. Clinical specimens were obtained from adult patients with B-cell acute lymphoblastic leukemia (B-ALL) (Supplementary Table 1). All human subjects provided informed consent, and the study was approved by the University of Southern California institutional review board. Mononuclear cells (MNC) were isolated by density centrifugation using Ficoll Paque Plus, density 1.077 (GE Healthcare Bio-Sciences) followed by two washes with Iscove's Modified Dulbecco's Medium (IMDM) (Thermo Fisher Scientific) and were frozen for later uses. During recovery, frozen cells were thawed and cultured in IMDM supplemented with 20\% fetal bovine serum (FBS) (VWR Life Science Seradigm) for 1 to 2 hours at $37^{\circ} \mathrm{C}$. Cells were then stained, analyzed, and sorted.

Leukemia cell culture and lentiviral transduction. Human B-ALL cells were sorted for human CD45 and CD19 from cryopreserved samples (Supplementary Table 2). These cells were either primary human bone marrow aspirates (ALL04, ALL06 and ALL20) or passaged mouse spleen cells (JFK88 and JFK93). Cells were cultured in StemSpan ${ }^{\mathrm{TM}}$ Serum-Free Expansion Medium II (SFEM II) (Stem Cell Technologies) in the presence of $20 \mathrm{ng} / \mathrm{ml}$ human FLT-3 ligand, $20 \mathrm{ng} / \mathrm{ml}$ human Interleukin-3 (IL-3) and 50 ng/ml human Stem Cell Factor (SCF) (all from Gibco by Life Technologies). After 24 hours of pre-stimulation under the culture condition, cells were washed and incubated for another 16 hours in the same medium with the addition of lentivirus carrying the DNA barcodes. $8 \mathrm{ng} / \mu$ polybrene was added to the culture to facilitate viral transduction. BALL cells were washed three times with Dulbecco's Phosphate Buffered Saline (D-PBS) (Gibco 
by Life Technologies) prior to transplantation. Transduction rate was generally below $30 \%$ in order to ensure that each cell was marked by a single barcode.

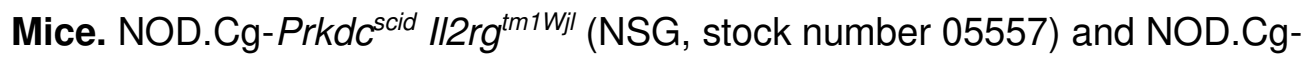
Prkdc ${ }^{\text {scid }} / 12$ rg $^{\text {tm1Wjl }}$ Tg (CMV-IL3,CSF2,KITLG)1Eav/MloySzJ (NSG-SGM3, stock number 013062) mice were obtained from Jackson Laboratory. Mice were bred and maintained at the Research Animal Facility of the University of Southern California. Animal procedures were approved by the Institutional Animal Care and Use Committee.

Human B-ALL engraftment. NSG or NSG-SGM3 mice were irradiated with 150 cGy and transplanted with 100,000 to 200,000 human B-ALL cells via tail vein injection. Mice were monitored daily for evidence of distress and were euthanized when human chimerism exceeded $90 \%$ of total MNC. At the end time point of each experiment, mouse peripheral blood was collected via perfusion using D-PBS with $10 \mathrm{mM}$ Ethylenediaminetetraacetic acid (EDTA) (Sigma-Aldrich). Spleen, bones, and tissues with noticeable extramedullary expansion were collected. Single cell suspensions were prepared by crushing the tissues in D-PBS with $2 \%$ FBS and filtered through a 70uM cell strainer. Cells were analyzed by flow cytometry. 500,000 barcoded cells were sorted for barcode analysis. Additional unsorted cells were frozen in IMDM with 20\% FBS and 10\% dimethylsulfoxide (DMSO) (Sigma-Aldrich) for later use.

Blood sample collection and FACS analysis. Blood samples were collected into D-PBS containing $10 \mathrm{mM}$ EDTA via a small transverse cut in the tail vein. $2 \%$ dextran (Pharmacosmos) was added to remove red blood cells. To further eliminate red blood cells, the remaining blood cells were treated with ammonium-chloride-potassium lysis buffer on ice for 5 minutes. After a 45-60-minute antibody incubation on ice, samples were suspended in D-PBS with $2 \%$ FBS and 4,6-Diamidino-2-phenylindole to distinguish dead cells. Cells were analyzed and sorted using the FACS-Aria cell sorters. Antibodies were obtained from eBioscience (currently Life Technologies/Thermo Fisher) and BioLegend (Supplementary Table 2). Flow cytometry data were analyzed using Diva software 8.0.1 (BD Biosciences).

Chemotherapy treatment. Barcoded human B-ALL cells were transplanted into sub lethally irradiated NSG or NSG-SGM3 mice. Once the human leukemia cell contribution reached 20$40 \%$ of total MNCs, mice were randomized and placed into one of the five chemotherapy groups: (i) vehicle control, (ii) combination therapy consisting of short-term intensive therapy 
followed by long-term maintenance therapy, (iii) short-term intensive therapy, (iv) prolonged intensive therapy, and (v) maintenance therapy. The intensive therapy consisted of Vincristine (Hospira Pharmaceuticals) $(0.25 \mathrm{mg} / \mathrm{kg}$ ) administered weekly via intravenous (IV) injection, Dexamethasone (AuroMedics Pharma) $(7.5 \mathrm{mg} / \mathrm{kg}$ ) administered Monday, Wednesday, and Friday via intraperitoneal (IP) injection, and L-asparaginase (Sigma-Tau Pharmaceuticals) (100 $\mathrm{IU} / \mathrm{kg}$ ) administered bi-weekly via IP injection. Maintenance therapy consisted of weekly Methotrexate (Accord Healthcare) $(5 \mathrm{mg} / \mathrm{kg})$ administered via IV or intramuscular injection. Vehicle group received weekly IV injections of Bacteriostatic Water (Hospira Pharmaceuticals) during the life span of the mice. The short-term intensive therapy group only received four weeks of intensive therapy. The combination therapy group received four weeks of intensive therapy followed with maintenance therapy. The prolonged intensive therapy group was treated with intensive therapy to the toleration of the mice as assessed by changes to their body weight (Supplementary Fig. 8). Toxicity was assessed based on body weight change. The range of prolonged intensive therapy treatment was 7 weeks for ALL04 and ALL20, and 10 weeks for ALL06. The maintenance therapy group was treated with maintenance therapy continuously during the life span of the mice. The range of maintenance therapy treatment was 5 weeks for ALL20, 14 weeks for ALL04, and 28 weeks for ALL06.

Leukemia progression was monitored throughout the duration of treatment by analyzing the peripheral blood. In addition, mouse weight was monitored weekly throughout treatment to assess therapy toxicity. When body weight dropped more than $20 \%$ of the starting weight, chemotherapy doses were adjusted. Animal care was in accordance with institutional guidelines. Survival (Kaplan-Meier) curves were generated using GraphPad Prism Software (San Diego, California) with significance determined by the log-rank test.

DNA barcode extraction and analysis. Genomic DNA was extracted from sorted barcoded leukemia cells and amplified using Phusion PCR master mix (Thermo Scientific, Waltham, MA). The PCR reactions were halted once they had progressed halfway through the exponential phase. PCR product was purified and analyzed using high-throughput sequencing. We combined sequencing data with FACS data to calculate the clonal abundance for each clone as indicated below. Clones with a clonal abundance greater than $0.01 \%$ were used for further analyses.

Clonal abundance \%

$$
=100 *\left[\frac{\# \text { of reads for each barcode }}{\text { total reads for all barcodes }}\right]\left[\frac{\# \text { of human cells }}{\text { total MNC }}\right]\left[\frac{\# \text { of GFP cells }}{\text { total human cells }}\right]
$$


Single cell RNA sequencing and data analysis. Single cell RNA sequencing (scRNA-seq) was performed following the manufacturer's protocol for the Chromium Single Cell 3' Library (10X Genomics, V2) with minor modifications as follows. Barcoded ALL04 and ALL20 cells (hCD45+GFP+) were sorted and loaded into the Chromium chip. After cDNA amplification, half of the amplified cDNA was used for the downstream fragmentation, adaptor ligation, and sample index PCR. The other half of the amplified cDNA was used to PCR amplify molecules that contain both the genetic tracking barcodes and the Chromium cellular barcodes. These molecules were then sequenced using the SMRT sequencing platform (Sequel II, Pacific Biosciences) to map genetic tracking barcodes to Chromium cellular barcodes, and thereby connecting clonal tracking data and gene expression data from the same cell. The cDNA libraries were sequenced using an Illumina HiSeq 4000 at a coverage of 50,000 raw reads per cell (paired end; read1: 26 cycles; 7 index: 8 cycles; read 2: 98 cycles). Raw data were processed using the Cell Ranger pipeline (10X Genomics, $v$ 2.1.0) for cellular barcode assignment and unique molecule identifier (UMI) quantification. From three 10X channels where we loaded the ALL04 and ALL20 cells, we recovered 4402, 5185, and 5542 cells, respectively. Cells with more than $10 \%$ UMls mapped to mitochondrial genes were excluded. After the filtering, the remaining cell numbers are 4291, 5093, and 5385, respectively. Genes with more than $2 \mathrm{UMIs}$ in more than $5 \%$ of cells were used for downstream analyses. Expression values for gene $i$ in cell $j$ were calculated by dividing UMI count values for gene $i$ by the sum of the UMI counts in cell $j$, and then multiplying by 10,000 to create transcripts per million (TPM) like values, and finally calculating $\log _{2}(T P M+1)$ as gene expression values. For comparing single cell gene expression data, $\mathrm{P}$ values were calculated using the one-sided Mann Whitney U-test.

False positive score calculation. False Positive Score (FPS) was calculated by comparing experimental data and scramble data. Five sets of scramble data were generated by randomly mapping tracking barcode data to gene expression data. For each gene, $P$ values were calculated by the one-sided Mann Whitney U-test using both the experimental data and the scramble data. FPS for each $P$ value of the experimental data was then calculated as the number of genes whose $P$ values were equal or smaller than this $P$ value in the scramble data (median of the five sets) divided by that gene number in the experimental data. Genes with FPS $<0.05$ and $P$ value $<0.05$ were considered significant. 
Statistical analysis. Diversity of clones was calculated using the Shannon Diversity Index, as implemented by Python package skbio.diversity (scikit-bio). K-Clustering method (Fig. 3b) was performed as implemented by python package sklearn.cluster.Kmeans (scikit-learn), the number of clusters was set to 2, and all other parameters were left as default. Tissue biases (Fig. $1 \mathrm{i}$ and $2 \mathrm{~d}$ ) were determined based on clonal abundance variations between blood and spleen (99\% confidence interval), assuming non-biased clones have equal abundance across tissues. P-values for clonal response across treatment groups were calculated via independent t-test with equal variance assumed. Significance in all figures was indicated as follows:

${ }^{* * *} P<0.001 ;{ }^{* *} P<0.01 ;{ }^{*} P<0.05 ;$ n.s. $P>0.05$.

Data Availability. Raw data and counts matrices are available at the GEO (GSE162506). Code Availability. Python scripts are available upon request.

\section{Acknowledgements}

The authors thank: USC Stem Cell Flow Cytometry Facility and CHLA Sequencing Core for technical support, C. Lytal for editing the text, and Q. Liu for manuscript discussion. R. L. is a Scholar of the Leukemia \& Lymphoma Society and a Richard N. Merkin Assistant Professor. H.C.-T. was supported by National Cancer Institute grant F31CA206463. M. C. V.-R. and B.G. were supported by California Institute for Regenerative Medicine grant EDUC2-08381. A. A. was supported by the University of Southern California Provost's Undergraduate Research Fellowship and the Rose Hills Research Fellowship. The project described was supported in part by award number P30CA014089 from the National Cancer Institute. The content is solely the responsibility of the authors and does not necessarily represent the official views of the National Cancer Institute or the National Institutes of Health.

\section{Author contributions}

R.L. conceptualized the project, designed the experiments, and wrote the manuscript. A.A.M. provided clinical advice, patient samples and helped design chemotherapy experiments. H.C.-T. designed, performed the experiments and wrote the manuscript. A.J., M.C.V.-R., A.A., C.S.B., B.G, and A.N. assisted with experiments. R.L., H.C.-T., J.E. and S.A. performed data analysis and generated figures. D.J. performed the single cell RNA sequencing experiments and relevant 
data analysis. M.M., G.X., J.-W.L., L.N.C., and L.H. provided patient samples. A.A.M., M.M. and A.N. edited the manuscript.

\section{Competing interests}

The authors declare no competing financial interests.

\section{Figure legends}

Fig. 1 Distinct leukemia clones expanded in extramedullary organs. a Clonal abundance was assessed in different tissues and organs and mapped to single cell RNA sequencing data. b The fractions of mouse cells, non-barcoded human leukemia cells (GFP-), and barcoded human leukemia cells (GFP+) in the mononuclear cells (MNC) of the peripheral blood from a xenografted mouse over time. c-e Representative images showing extramedullary sites of leukemia expansion. Quarters are shown in (d) as size references. Additional mice are shown in Supplementary Fig. 2. f-h Clonal distribution across different tissues and organs in representative mice. Each color represents one distinct genetic barcode corresponding to a leukemia clone. Additional mice are shown in Supplementary Fig. 3, 4c, and 4f. i Comparison of clonal abundance between the peripheral blood and the spleen or the ovary. Markers of the same shape represent data from one mouse. $99 \%$ confidence intervals of correlation were determined by the blood and spleen comparison and highlighted by dashed grey lines. $\mathbf{j}$ The CMC2 gene was significantly upregulated in clones more abundant in the ovary than clones more abundant in the blood. Black bar indicates the mean, and white dot represents the median.

\section{Fig. 2 Spatially confined expansion of leukemia clones in the bone marrow of primary} recipients. a-b Heatmaps showing Pearson Correlations of clonal abundance across different tissues and organs. Each column corresponds to one primary recipient mouse. The clonal diversity of each mouse is shown below, where each color represents a unique clone. a Comparison between the peripheral blood, spleen and bone marrow (BM) from the spine. $\mathbf{b}$ Comparison of the bone marrow cells harvested from three distinct anatomical regions: left leg, right leg, and spine. c Leukemia clonal diversity across different tissues and organs from one representative mouse. Additional mice are shown in Supplementary Fig. 4. Each color 
represents one distinct genetic barcode corresponding to a leukemia clone. $\mathbf{d}$ Comparison of clonal abundance between the peripheral blood and the spleen or the leg BM for ALL04 clones. Markers of the same shape represent data from one mouse. 99\% confidence intervals were determined by the blood and spleen comparison and highlighted by dashed lines. e Genes significantly differentially expressed in ALL04 clones more abundant in the BM compared to clones more abundant in the blood. Black bar indicates the mean, and white dot represents the median. MNC - mononuclear cells.

Fig. 3 Clonal selection occurs during serial transplantation. a Barcoded B-ALL cells were consecutively transplanted through three generations of mice. Clonal abundance was assessed in each recipient. $\mathbf{b}$ K-means clustering separated leukemia clones into diminishing and expanding groups. Each section in the column represents average abundance of each clone. c Human B-ALL cells were barcoded before primary transplantation and transplanted into multiple secondary recipients. d Clonal abundance changes during serial transplantation. Each color represents a genetic barcode corresponding to a leukemia clone. Each column shows data from one mouse. Additional mice are shown in Supplementary Fig. 7. e Clonal abundance comparison between the primary and secondary recipients during serial transplantation. Each dot represents a barcoded clone, and each color depicts one primary mouse. MNC mononuclear cells. $\mathbf{f}$ Pearson correlation of clonal abundance between different mice during serial transplantation. ${ }^{* *} \mathrm{P}<0.001$. All clonal abundance data in Fig. 3 originates from spleen cell analyses. Barcoded spleen cells were transplanted during serial transplantation.

\section{Fig. 4 Comparing the same leukemia clones during different chemotherapy treatments} across multiple mice. a Kaplan-Meier survival plots of PDX mice under various treatments. Color bars illustrate the duration of the treatment for each group. $\mathbf{b}$ Human chimerism in the peripheral blood. Shown are the means of all experimental mice. Mouse variations are provided in Supplementary Fig. 9. c Clonal abundance in mouse blood before treatments and at the end time point for each mouse. Each column represents one mouse. Each color represents one distinct genetic barcode corresponding to a leukemia clone. d-e Representative clonal dynamics during combination therapy in two PDX mice. Additional mice are shown in Supplementary Figs. 10-12. The black colored clone in multiple mice of (c-d) and Supplementary Fig. 11 represents non-barcoded (GFP-) human cells from patient ALL06. PCR analysis reveals that these GFPhuman cells did not carry any genetic barcode (Supplementary Fig. 13). They may have 
originated from a clone that had lost the barcode construct or that may have been collected due to a sorting error. These non-barcoded human cells were not detected in mice that received the vehicle treatment or short intensive therapy, or in any mice that received sorted GFP+ ALL cells derived from the other patient samples.

\section{Fig. 5 Some B-ALL clones respond differentially to intensive and maintenance} chemotherapy in distinct but consistent manners across multiple mice. a Comparing chemotherapy response of the same clones in different tissues. Shown are the $\log _{2}$ fold differences between the average clonal abundances of two chemotherapy treatments for each tissue. R - Pearson's correlation coefficient. Each dot represents one clone. b Example clones that responded differently under various chemotherapy treatments. Each marker represents data from one tissue and one mouse. $\mathrm{c}$ left, ALL04 clones that responded better to combination therapy than to intensive therapy. $\mathbf{d}$ left, ALL04 clones that responded better to maintenance therapy than to intensive therapy. e left, ALL20 clones that responded better to intensive therapy than to maintenance therapy. c-e right, Genes significantly differentially expressed in the clones shown on the left (blue) compared to all other clones from the same patient samples (orange). Black bar indicates the mean, and white dot represents the median. BM - bone marrow, MNC - mononuclear cells.

Fig. 6 Relapsed leukemia cells generate greater clonal dominance in the PDX model than chemotherapy-naïve cells from the same patients. a Human B-ALL cells acquired from two patients at distinct disease stages (chemotherapy naïve and chemotherapy relapsed) were genetically labeled with DNA barcodes and transplanted into irradiated immunocompromised mice. Leukemia progression was monitored through peripheral blood analysis. b Human chimerism of the mouse peripheral blood following transplantation of diagnostic (naïve) and relapsed leukemia cells from the same patients. Each line depicts data from one mouse. c Number of unique DNA barcodes detected in the peripheral blood. Each line depicts data from one mouse. ${ }^{*} \mathrm{P}<0.01$. $\mathbf{d}$ Clonal diversity in the peripheral blood over time. Each line represents data from one recipient mouse. ${ }^{*} \mathrm{P}<0.05$. e Clonal abundance distribution changes over time. Histogram shows data from all mice. $\mathbf{f}$ Clonal composition in the peripheral blood of mice xenografted by naïve and relapsed samples of the same patient. Each color represents one distinct genetic barcode corresponding to a leukemia clone. Additional mice are shown in Supplementary Fig. 25. g Clonal abundance change over time. Each line represents one clone. 
Each color depicts one mouse. Shown are all clones exceeding $1 \%$ of mononuclear cells (MNC) abundance at any time point.

\section{References}

1. Burrell, R. A., McGranahan, N., Bartek, J. \& Swanton, C. The causes and consequences of genetic heterogeneity in cancer evolution. Nature 501, 338-345 (2013).

2. Shackleton, M., Quintana, E., Fearon, E. R. \& Morrison, S. J. Heterogeneity in cancer: cancer stem cells versus clonal evolution. Cell 138, 822-9 (2009).

3. McGranahan, N. \& Swanton, C. Clonal Heterogeneity and Tumor Evolution: Past, Present, and the Future. Cell 168, 613-628 (2017).

4. Almendro, V., Marusyk, A. \& Polyak, K. Cellular Heterogeneity and Molecular Evolution in Cancer. Annu Rev Pathology Mech Dis 8, 277-302 (2013).

5. Esteller, M. Epigenetics in Cancer. New Engl J Medicine 358, 1148-1159 (2008).

6. Greaves, M. \& Maley, C. C. Clonal evolution in cancer. Nature 481, 306-313 (2012).

7. Kreso, A. \& Dick, J. E. Evolution of the cancer stem cell model. Cell stem cell 14, 275-91 (2014).

8. Mazor, T., Pankov, A., Song, J. S. \& Costello, J. F. Intratumoral Heterogeneity of the Epigenome. Cancer Cell 29, 440-451 (2016).

9. Meacham, C. E. \& Morrison, S. J. Tumour heterogeneity and cancer cell plasticity. Nature 501, 328-37 (2013).

10. Kreso, A. et al. Variable clonal repopulation dynamics influence chemotherapy response in colorectal cancer. Science (New York, N.Y.) 339, 543-8 (2013).

11. Dagogo-Jack, I. \& Shaw, A. T. Tumour heterogeneity and resistance to cancer therapies. Nat Rev Clin Oncol 15, 81-94 (2018).

12. Chabon, J. J. et al. Circulating tumour DNA profiling reveals heterogeneity of EGFR inhibitor resistance mechanisms in lung cancer patients. Nat Commun 7, 11815 (2016).

13. Sequist, L. V. et al. Genotypic and Histological Evolution of Lung Cancers Acquiring Resistance to EGFR Inhibitors. Sci Transl Med 3, 75ra26-75ra26 (2011). 
14. Gorre, M. E. et al. Clinical Resistance to STI-571 Cancer Therapy Caused by BCR-ABL Gene Mutation or Amplification. Science 293, 876-880 (2001).

15. McGranahan, N. \& Swanton, C. Biological and Therapeutic Impact of Intratumor Heterogeneity in Cancer Evolution. Cancer Cell 27, 15-26 (2015).

16. Darmanis, S. et al. Single-Cell RNA-Seq Analysis of Infiltrating Neoplastic Cells at the Migrating Front of Human Glioblastoma. Cell Reports 21, 1399-1410 (2017).

17. Paguirigan, A. L. et al. Single-cell genotyping demonstrates complex clonal diversity in acute myeloid leukemia. Science translational medicine 7, $281 \mathrm{re2}$ (2015).

18. Melchor, L., Brioli, A., Wardell, C., Murison, A. \& Potter, N. Single-cell genetic analysis reveals the composition of initiating clones and phylogenetic patterns of branching and parallel evolution in myeloma. (2014).

19. Gawad, C., Koh, W. \& Quake, S. R. Dissecting the clonal origins of childhood acute lymphoblastic leukemia by single-cell genomics. Proceedings of the National Academy of Sciences of the United States of America 111, 17947-52 (2014).

20. Kim, C. et al. Chemoresistance Evolution in Triple-Negative Breast Cancer Delineated by Single-Cell Sequencing. Cell 173, 879-893.e13 (2018).

21. Baslan, T. \& Hicks, J. Unravelling biology and shifting paradigms in cancer with single-cell sequencing. Nat Rev Cancer 17, 557-569 (2017).

22. Puram, S. V. et al. Single-Cell Transcriptomic Analysis of Primary and Metastatic Tumor Ecosystems in Head and Neck Cancer. Cell 171, 1611-1624.e24 (2017).

23. Cresswell, G. D. et al. Mapping the breast cancer metastatic cascade onto ctDNA using genetic and epigenetic clonal tracking. Nat Commun 11, 1446 (2020).

24. Ding, L. et al. Clonal evolution in relapsed acute myeloid leukaemia revealed by wholegenome sequencing. Nature 481, 506-10 (2012).

25. Jan, M. et al. Clonal evolution of preleukemic hematopoietic stem cells precedes human acute myeloid leukemia. Science translational medicine 4, 149ra118 (2012).

26. Wang, Y. et al. Clonal evolution in breast cancer revealed by single nucleus genome sequencing. Nature 512, 155-160 (2014).

27. Eirew, P. et al. Dynamics of genomic clones in breast cancer patient xenografts at singlecell resolution. Nature 518, 422-6 (2015). 
28. Klco, J. M. et al. Functional Heterogeneity of Genetically Defined Subclones in Acute Myeloid Leukemia. Cancer Cell 25, 379-392 (2014).

29. Gerlinger, M. et al. Intratumor Heterogeneity and Branched Evolution Revealed by Multiregion Sequencing. New Engl J Med 366, 883-892 (2012).

30. Miles, L. A. et al. Single-cell mutation analysis of clonal evolution in myeloid malignancies. Nature 587, 477-482 (2020).

31. Hyo-eun, C., Ruddy, D. \& Radhakrishna, V. Studying clonal dynamics in response to cancer therapy using high-complexity barcoding. Nature Medicine (2015) doi:10.1038/nm.3841.

32. Merino, D. et al. Barcoding reveals complex clonal behavior in patient-derived xenografts of metastatic triple negative breast cancer. Nat Commun 10, 766 (2019).

33. Nguyen, L. V. et al. DNA barcoding reveals diverse growth kinetics of human breast tumour subclones in serially passaged xenografts. Nature communications 5, 5871 (2014).

34. Cassidy, J. W., Caldas, C. \& Bruna, A. Maintaining Tumor Heterogeneity in Patient-Derived Tumor Xenografts. Cancer Res 75, 2963-2968 (2015).

35. Byrne, A. T. et al. Interrogating open issues in cancer precision medicine with patientderived xenografts. Nature Reviews Cancer (2017) doi:10.1038/nrc.2016.140.

36. Lu, R., Neff, N. F., Quake, S. R. \& Weissman, I. L. Tracking single hematopoietic stem cells in vivo using high-throughput sequencing in conjunction with viral genetic barcoding. Nature biotechnology 29, 928-33 (2011).

37. Bramlett, C. et al. Clonal tracking using embedded viral barcoding and high-throughput sequencing. Nat Protoc 15, 1436-1458 (2020).

38. Brewer, C., Chu, E., Chin, M. \& Lu, R. Transplantation Dose Alters the Differentiation Program of Hematopoietic Stem Cells. Cell reports 15, 1848-57 (2016).

39. Nguyen, L. et al. Functional compensation between hematopoietic stem cell clones in vivo. EMBO reports 19, (2018).

40. Lu, R., Czechowicz, A., Seita, J., Jiang, D. \& Weissman, I. L. Clonal-level lineage commitment pathways of hematopoietic stem cells in vivo. Proceedings of the National Academy of Sciences of the United States of America 116, 1447-1456 (2019).

41. Macosko, E. Z. et al. Highly Parallel Genome-wide Expression Profiling of Individual Cells Using Nanoliter Droplets. Cell 161, 1202-1214 (2015). 
42. Zheng, G. X. Y. et al. Massively parallel digital transcriptional profiling of single cells. Nat Commun 8, 14049 (2017).

43. Kersseboom, R. et al. Bruton's Tyrosine Kinase Cooperates with the B Cell Linker Protein SLP-65 as a Tumor Suppressor in Pre-B Cells. J Exp Medicine 198, 91-98 (2003).

44. Feldhahn, N. et al. Deficiency of Bruton's tyrosine kinase in B cell precursor leukemia cells. P Natl Acad Sci Usa 102, 13266-13271 (2005).

45. Szymanska, B. et al. Pharmacokinetic Modeling of an Induction Regimen for In Vivo Combined Testing of Novel Drugs against Pediatric Acute Lymphoblastic Leukemia Xenografts. PLOS ONE 7, e33894 (2012).

46. Park, E. et al. Targeting survivin overcomes drug resistance in acute lymphoblastic leukemia. Blood 118, 2191-9 (2011).

47. Jorfi, S. et al. Inhibition of microvesiculation sensitizes prostate cancer cells to chemotherapy and reduces docetaxel dose required to limit tumor growth in vivo. Scientific reports 5, 13006 (2015).

48. Tijchon, E. et al. Tumor suppressors BTG1 and BTG2 regulate early mouse B-cell development. Haematologica 101, e272-e276 (2016).

49. Islaih, M. et al. Relationships between genomic, cell cycle, and mutagenic responses of TK6 cells exposed to DNA damaging chemicals. Mutat Res Fundam Mol Mech Mutagen 578, 100116 (2005).

50. Chen, J.-G., Yang, C.-P., Cammer, M. \& Horwitz, S. Gene expression and mitotic exit induced by microtubule-stabilizing drugs. Cancer Res 63, 7891-9 (2003).

51. Delgado, J., Bustos, J. G., Jimenez, M., Quevedo, E. \& Hernandez-Navarro, F. Are Activation Markers (CD25, CD38 and CD103) Predictive of Sensitivity to Purine Analogues in Patients with T-cell Prolymphocytic Leukemia and other Lymphoproliferative Disorders? Leukemia Lymphoma 43, 2331-2334 (2009).

52. Donk, N. W. van de et al. Monoclonal antibodies targeting CD38 in hematological malignancies and beyond. Immunol Rev 270, 95-112 (2016).

53. Nam, S. et al. A pathway-based approach for identifying biomarkers of tumor progression to trastuzumab-resistant breast cancer. Cancer Lett 356, 880-890 (2015). 
54. Bruna, A. et al. A Biobank of Breast Cancer Explants with Preserved Intra-tumor Heterogeneity to Screen Anticancer Compounds. Cell 167, 260-274.e22 (2016).

55. Stewart, E. et al. Orthotopic patient-derived xenografts of paediatric solid tumours. Nature 549, 96-100 (2017).

56. Ben-David, U. et al. Patient-derived xenografts undergo mouse-specific tumor evolution. Nat Genet 49, 1567-1575 (2017).

57. Hidalgo, M. et al. Patient-Derived Xenograft Models: An Emerging Platform for Translational Cancer Research. Cancer Discov 4, 998-1013 (2014).

58. Marangoni, E. et al. A New Model of Patient Tumor-Derived Breast Cancer Xenografts for Preclinical Assays. Clin Cancer Res 13, 3989-3998 (2007).

59. Gerstein, R. et al. Patient-Derived Xenografts (PDX) of B Cell Lymphoma in NSG Mice: A Mouse Avatar for Developing Personalized Medicine. Blood 126, 5408-5408 (2015).

60. Koga, Y. \& Ochiai, A. Systematic Review of Patient-Derived Xenograft Models for Preclinical Studies of Anti-Cancer Drugs in Solid Tumors. Cells 8, 418 (2019).

61. Lin, D. et al. High Fidelity Patient-Derived Xenografts for Accelerating Prostate Cancer Discovery and Drug Development. Cancer Res 74, 1272-1283 (2014).

62. Tentler, J. J. et al. Patient-derived tumour xenografts as models for oncology drug development. Nat Rev Clin Oncol 9, 338-350 (2012).

63. Sausville, E. A. \& Burger, A. M. Contributions of Human Tumor Xenografts to Anticancer Drug Development. Cancer Res 66, 3351-3354 (2006).

64. Fiebig, H. H., Maier, A. \& Burger, A. M. Clonogenic assay with established human tumour xenografts correlation of in vitro to in vivo activity as a basis for anticancer drug discovery. Eur $J$ Cancer 40, 802-820 (2004).

65. Brunning, R. D., Bloomfield, C. D., McKenna, R. W. \& Peterson, L. A. Bilateral Trephine Bone Marrow Biopsies in Lymphoma and Other Neoplastic Diseases. Ann Intern Med 82, 365 (1975).

66. Coller, B. S., Chabner, B. A. \& Gralnick, H. R. Frequencies and patterns of bone marrow involvement in non-hodgkin lymphomas: Observations on the value of bilateral biopsies. Am $J$ Hematol 3, 105-119 (1977). 
67. Ebinger, S. et al. Characterization of Rare, Dormant, and Therapy-Resistant Cells in Acute Lymphoblastic Leukemia. Cancer Cell 30, 849-862 (2016).

68. Kato, M. \& Manabe, A. Treatment and biology of pediatric acute lymphoblastic leukemia. Pediatr Int 60, 4-12 (2018). 


\section{Figures}

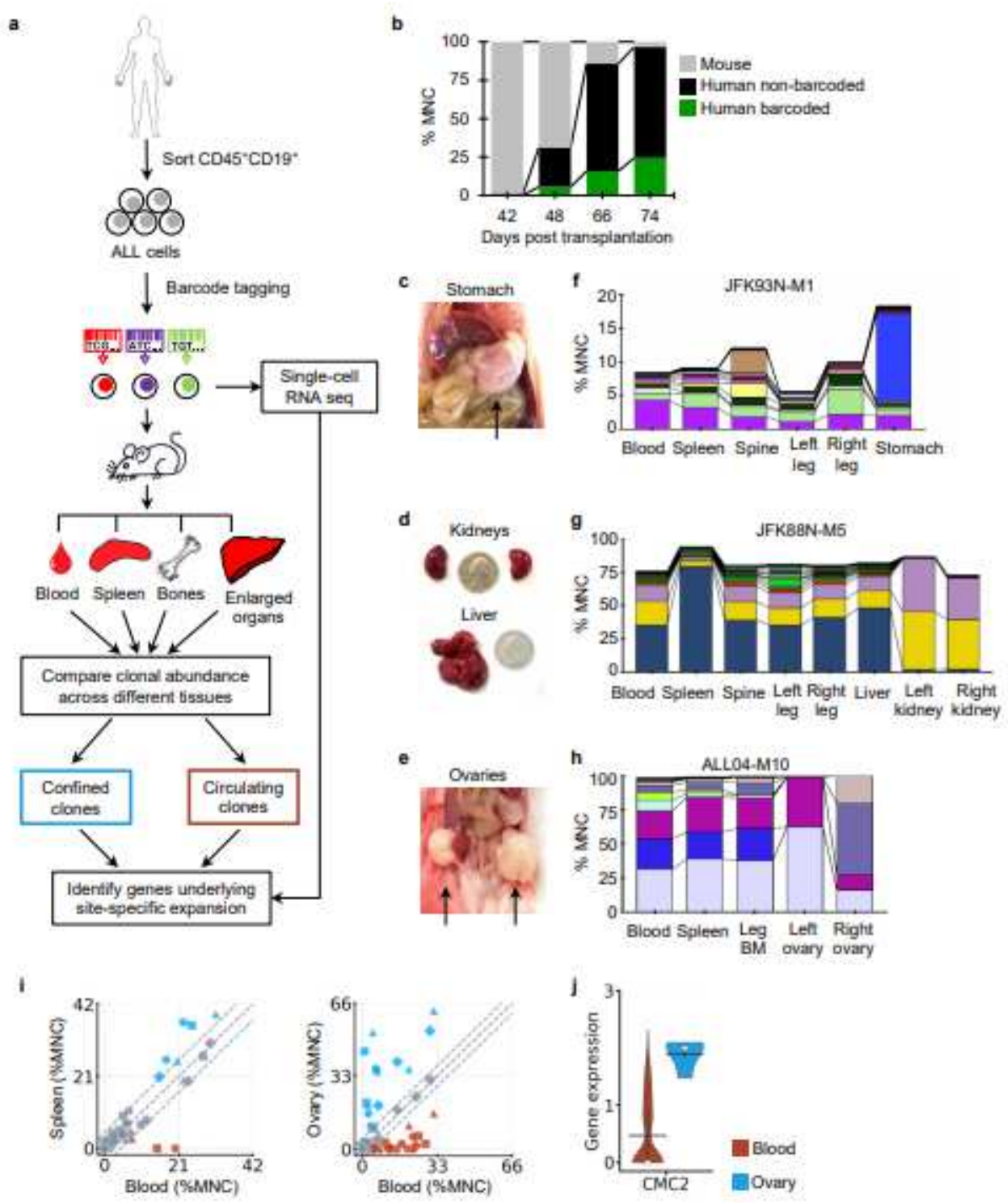

Figure 1

Distinct leukemia clones expanded in extramedullary organs. a Clonal abundance was assessed in different tissues and organs and mapped to single cell RNA sequencing data. $b$ The fractions of mouse cells, non-barcoded human leukemia cells (GFP-), and barcoded human leukemia cells (GFP+) in the mononuclear cells (MNC) of the peripheral blood from a xenografted mouse over time. c-e Representative images showing extramedullary sites of leukemia expansion. Quarters are shown in (d) as size references. Additional mice are shown in Supplementary Fig. 2. f-h Clonal distribution across different tissues and organs in representative mice. Each color represents one distinct genetic barcode corresponding to a leukemia clone. Additional mice are shown in Supplementary Fig. 3, 4c, and 4f. i Comparison of clonal abundance between the peripheral blood and the spleen or the ovary. Markers of the same shape represent data from one mouse. $99 \%$ confidence intervals of correlation were determined by the blood and spleen comparison and highlighted by dashed grey lines. j The CMC2 gene was 
significantly upregulated in clones more abundant in the ovary than clones more abundant in the blood. Black bar indicates the mean, and white dot represents the median.

a

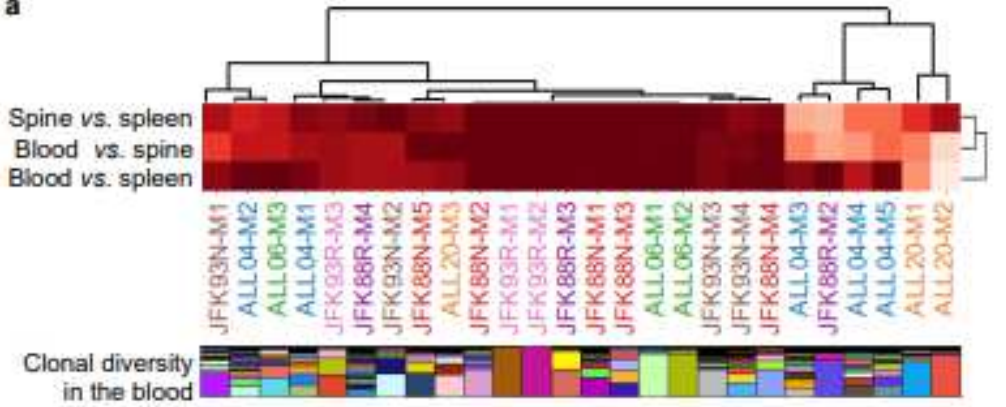

b

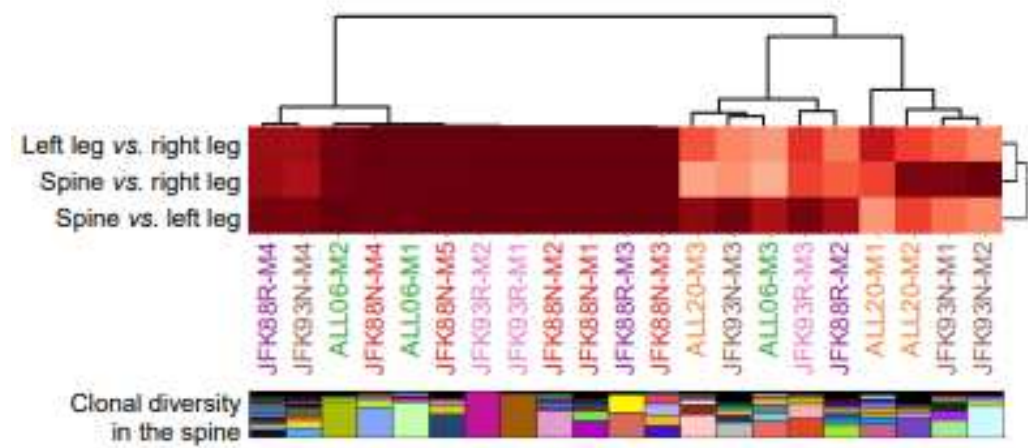

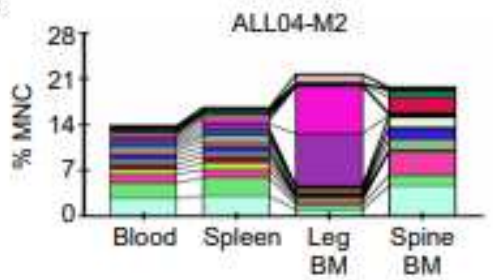

d
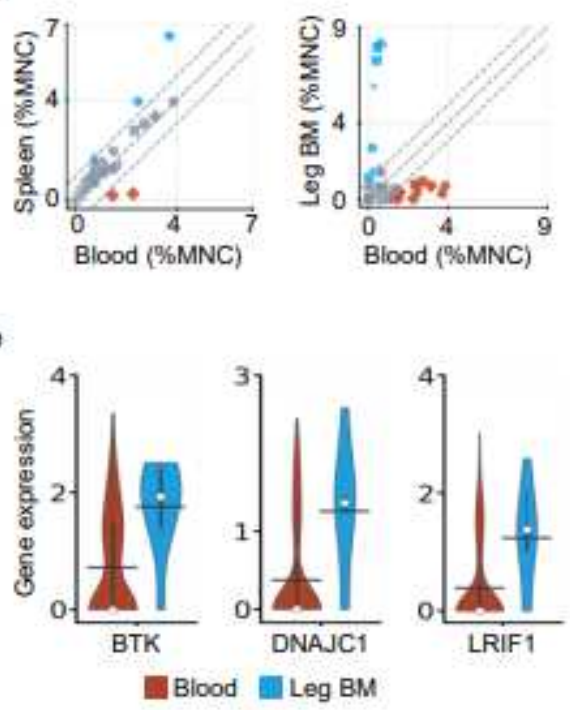

\section{Figure 2}

Spatially confined expansion of leukemia clones in the bone marrow of primary recipients. a-b Heatmaps showing Pearson Correlations of clonal abundance across different tissues and organs. Each column corresponds to one primary recipient mouse. The clonal diversity of each mouse is shown below, where each color represents a unique clone. a Comparison between the peripheral blood, spleen and bone marrow (BM) from the spine. b Comparison of the bone marrow cells harvested from three distinct anatomical regions: left leg, right leg, and spine. c Leukemia clonal diversity across different tissues and organs from one representative mouse. Additional mice are shown in Supplementary Fig. 4. Each color 16 represents one distinct genetic barcode corresponding to a leukemia clone. d Comparison of clonal abundance between the peripheral blood and the spleen or the leg BM for ALL04 clones. Markers of the same shape represent data from one mouse. $99 \%$ confidence intervals were determined by the blood and spleen comparison and highlighted by dashed lines. e Genes significantly differentially expressed in ALL04 clones more abundant in the BM compared to clones more abundant in the blood. Black bar indicates the mean, and white dot represents the median. MNC - mononuclear cells. 
a

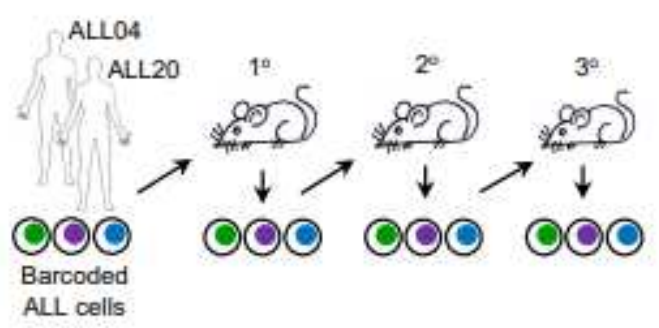

c ALLO4

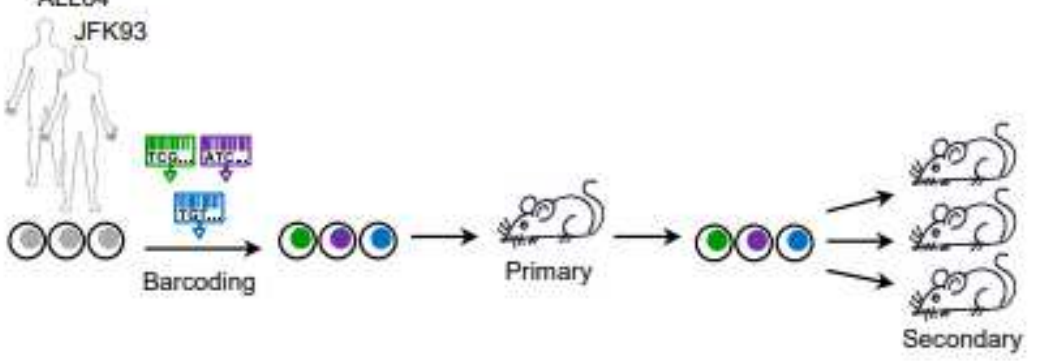

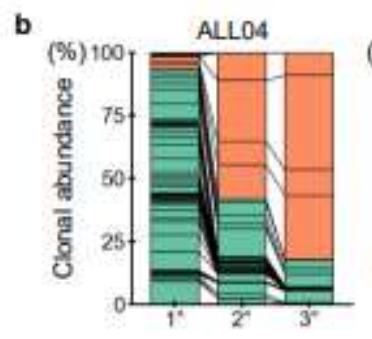

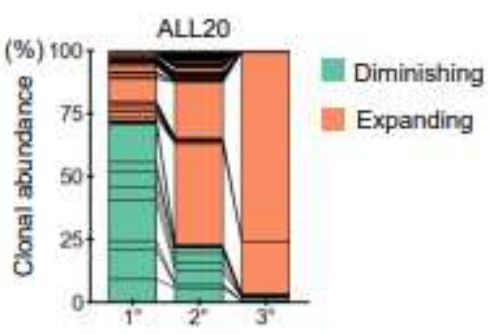

d

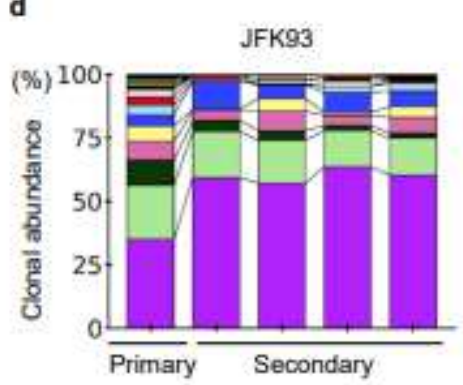

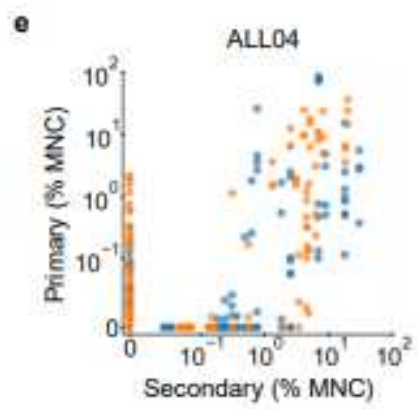
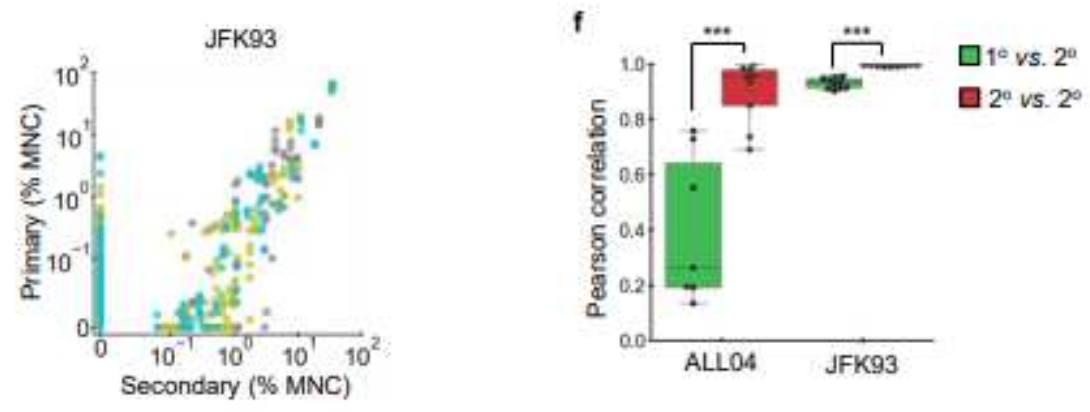

\section{Figure 3}

Clonal selection occurs during serial transplantation. a Barcoded B-ALL cells were consecutively transplanted through three generations of mice. Clonal abundance was assessed in each recipient. b Kmeans clustering separated leukemia clones into diminishing and expanding groups. Each section in the column represents average abundance of each clone. c Human B-ALL cells were barcoded before primary transplantation and transplanted into multiple secondary recipients. $\mathrm{d}$ Clonal abundance changes during serial transplantation. Each color represents a genetic barcode corresponding to a leukemia clone. Each column shows data from one mouse. Additional mice are shown in Supplementary Fig. 7. e Clonal abundance comparison between the primary and secondary recipients during serial transplantation. Each dot represents a barcoded clone, and each color depicts one primary mouse. MNC - mononuclear cells. $f$ Pearson correlation of clonal abundance between different mice during serial transplantation. ${ }^{\star} * \star \mathrm{P}<0.001$. All clonal abundance data in Fig. 3 originates from spleen cell analyses. Barcoded spleen cells were transplanted during serial transplantation. 
a

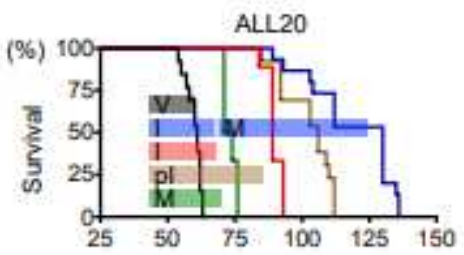

b

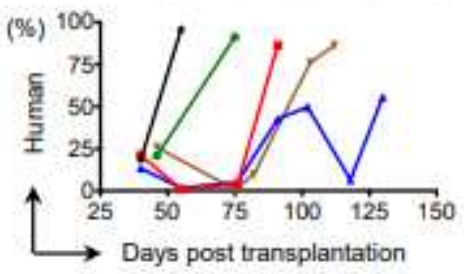

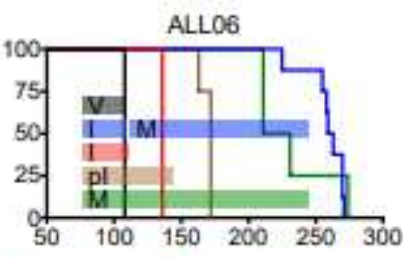

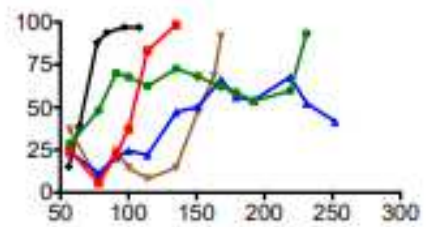

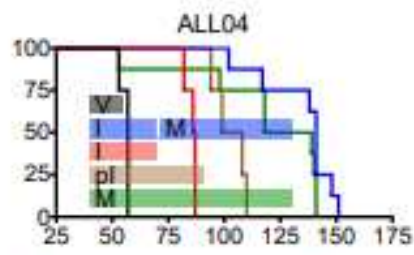

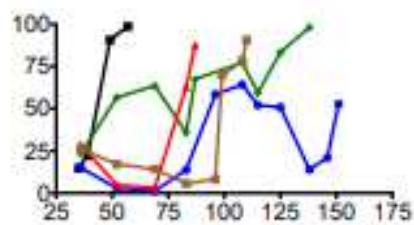

Colors apply to: a-c

- Vehicle (V) = Intensive + maintenance (IM) $\mathbf{n}$ Intensive (I)

arolonged intensive (pl)

Maintenance (M)

C

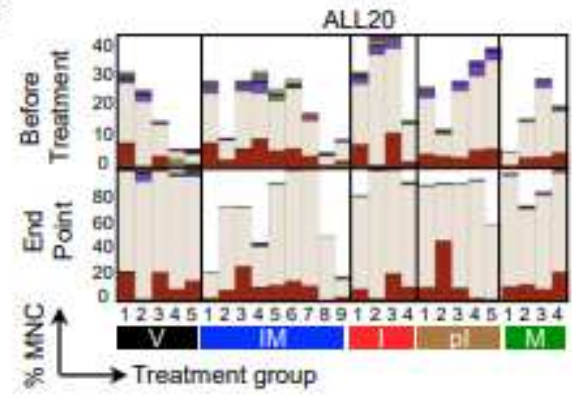

d

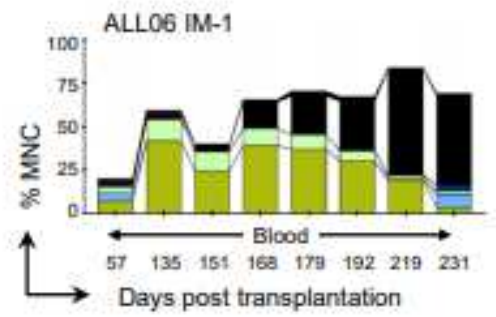

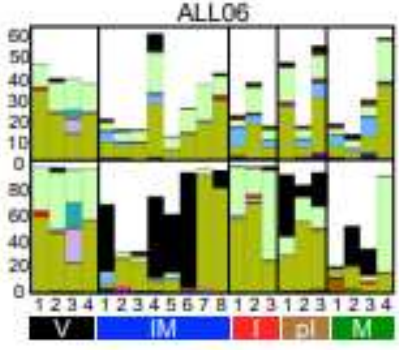

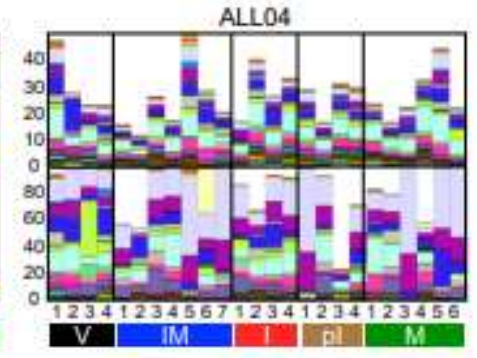

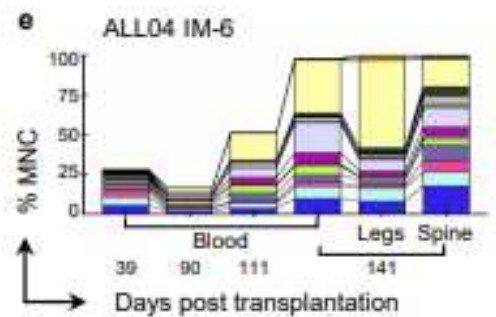

Figure 4

Comparing the same leukemia clones during different chemotherapy treatments across multiple mice. a Kaplan-Meier survival plots of PDX mice under various treatments. Color bars illustrate the duration of the treatment for each group. b Human chimerism in the peripheral blood. Shown are the means of all experimental mice. Mouse variations are provided in Supplementary Fig. 9. c Clonal abundance in mouse blood before treatments and at the end time point for each mouse. Each column represents one mouse. Each color represents one distinct genetic barcode corresponding to a leukemia clone. d-e Representative clonal dynamics during combination therapy in two PDX mice. Additional mice are shown in Supplementary Figs. 10-12. The black colored clone in multiple mice of (c-d) and Supplementary Fig. 11 represents non-barcoded (GFP-) human cells from patient ALL06. PCR analysis reveals that these GFPhuman cells did not carry any genetic barcode (Supplementary Fig. 13). They may have 17 originated from a clone that had lost the barcode construct or that may have been collected due to a sorting error. These non-barcoded human cells were not detected in mice that received the vehicle treatment or short intensive therapy, or in any mice that received sorted GFP+ ALL cells derived from the other patient samples. 

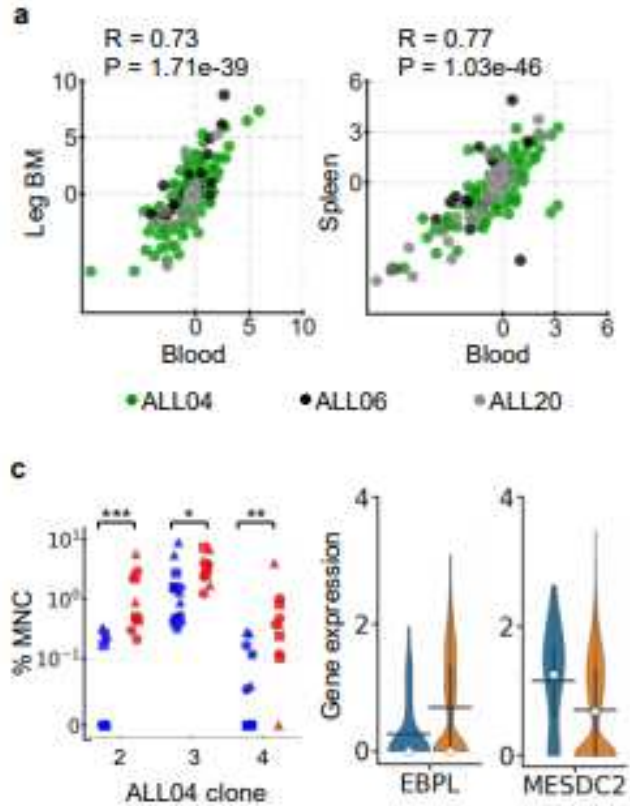

d

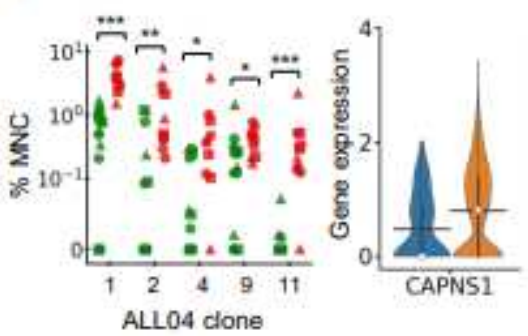

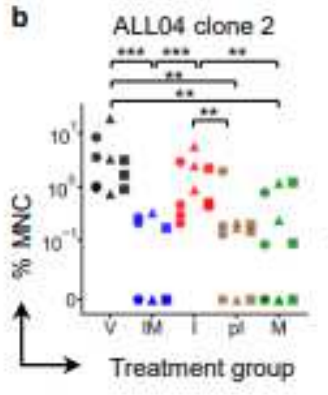
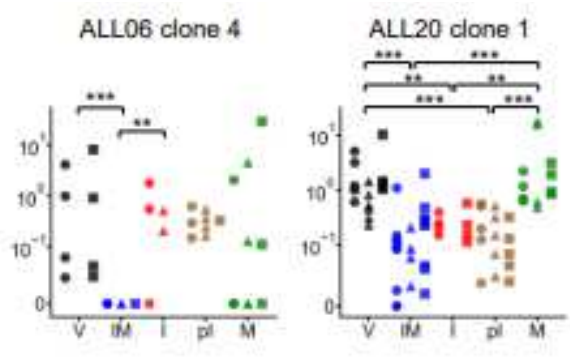

Colors and shapes apply to: b-e

- Vehicle $(V)$ Intensive + maintenance (IM) $=$ Intensive (I)

m Prolonged intensive (pl) $=$ Maintenance (M)

OBlood $\square$ Spleen $\triangle$ Bone Marrow

Colors apply to: $0-e$

Selected clones Rest of the clones

\section{Figure 5}

Some B-ALL clones respond differentially to intensive and maintenance chemotherapy in distinct but consistent manners across multiple mice. a Comparing chemotherapy response of the same clones in different tissues. Shown are the log2 fold differences between the average clonal abundances of two chemotherapy treatments for each tissue. R - Pearson's correlation coefficient. Each dot represents one clone. b Example clones that responded differently under various chemotherapy treatments. Each marker represents data from one tissue and one mouse. c left, ALL04 clones that responded better to combination therapy than to intensive therapy. $\mathrm{d}$ left, ALL04 clones that responded better to maintenance therapy than to intensive therapy. e left, ALL20 clones that responded better to intensive therapy than to maintenance therapy. c-e right, Genes significantly differentially expressed in the clones shown on the left (blue) compared to all other clones from the same patient samples (orange). Black bar indicates the mean, and white dot represents the median. BM - bone marrow, MNC - mononuclear cells. 


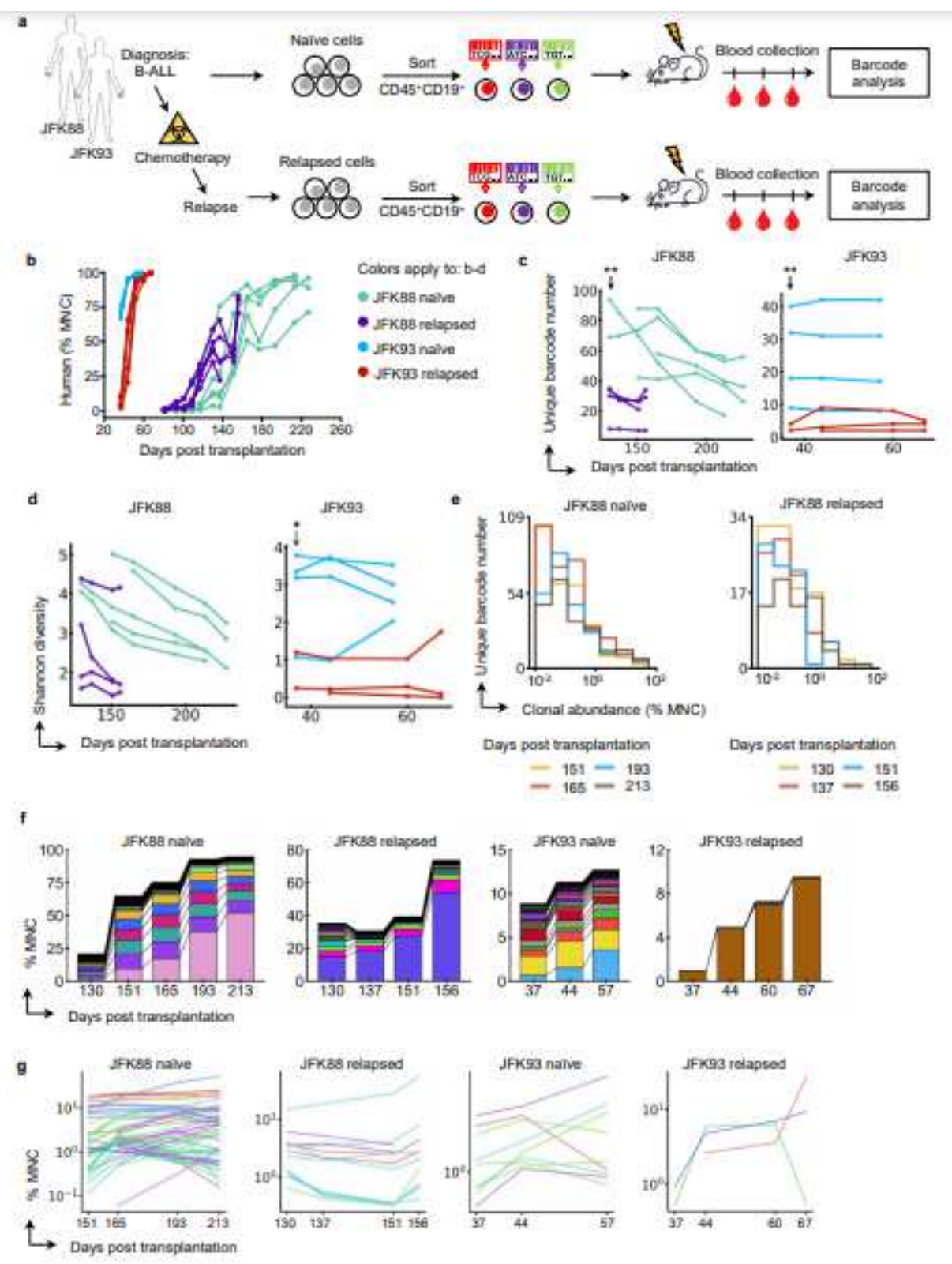

Figure 6

Relapsed leukemia cells generate greater clonal dominance in the PDX model than chemotherapy-naïve cells from the same patients. a Human B-ALL cells acquired from two patients at distinct disease stages (chemotherapy naïve and chemotherapy relapsed) were genetically labeled with DNA barcodes and transplanted into irradiated immunocompromised mice. Leukemia progression was monitored through peripheral blood analysis. b Human chimerism of the mouse peripheral blood following transplantation of diagnostic (naïve) and relapsed leukemia cells from the same patients. Each line depicts data from one mouse. c Number of unique DNA barcodes detected in the peripheral blood. Each line depicts data from one mouse. ${ }^{*} \mathrm{P}<0.01$. $\mathrm{d}$ Clonal diversity in the peripheral blood over time. Each line represents data from one recipient mouse. ${ }^{*} \mathrm{P}<0.05$. e Clonal abundance distribution changes over time. Histogram shows data from all mice. $f$ Clonal composition in the peripheral blood of mice xenografted by naïve and relapsed samples of the same patient. Each color represents one distinct genetic barcode corresponding 
to a leukemia clone. Additional mice are shown in Supplementary Fig. 25. g Clonal abundance change over time. Each line represents one clone. 18 Each color depicts one mouse. Shown are all clones exceeding $1 \%$ of mononuclear cells (MNC) abundance at any time point.

\section{Supplementary Files}

This is a list of supplementary files associated with this preprint. Click to download.

- ALLSupplementary121920NatComm.pdf 